\title{
Erratum:
}

\section{Thermodynamics of noninteracting bosonic gases in cubic optical lattices versus ideal homogeneous Bose gases}

\author{
[Int. J. Mod. Phys. B, Vol. 29, No. 18 (2015) 1550123 (25 pages)] \\ Abdulla Rakhimov*,† and Iman N. Askerzade ${ }^{\dagger, \ddagger}$ \\ * Institute of Applied Physics, National University of Uzbekistan, \\ Tashkent 100174, Uzbekistan \\ ${ }^{\dagger}$ Computer Engineering Department of Ankara University, Ankara 06100, Turkey \\ ¥ Institute of Physics, Azerbaijan National Academy of Sciences, \\ AZ1143 Baku, Azerbaijan \\ *rakhimovabd@yandex.ru
}

Published 22 December 2015

Figures on pages 1550123-8 (Fig. 2) and 1550123-11 (Fig. 3) should be replaced by following figures respectively.

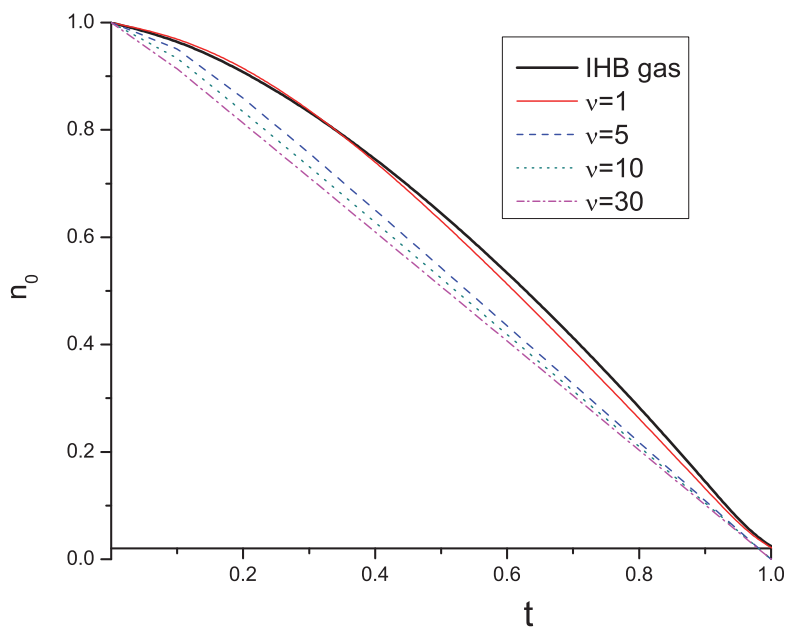

Fig. 2. (Color online) The condensed fraction $n_{0}$ versus reduced temperature $\tau=T / T_{c}^{0}$, as an exact solution of Eqs. (21) and (22) for different values of $\nu$. The thick solid line corresponds to the IHB gas given by (23). 
A. Rakhimov \& I. N. Askerzade

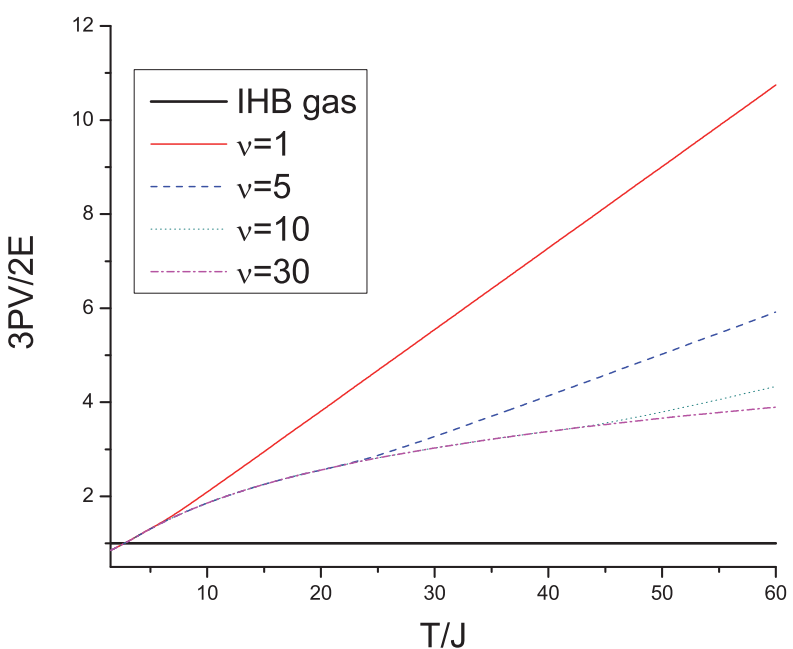

Fig. 3. (Color online) This relation between the internal energy and the thermodynamic potential, $|\Omega|$, equals exactly to unity for IHB gas, as presented by the thick solid line (see Eqs. (A.6) and (A.10)). 